\title{
New compact TEM-type deflecting and crabbing rf structure
}

\author{
J. R. Delayen* \\ Center for Accelerator Science, Old Dominion University, Norfolk, Virginia 23529, USA, \\ and Accelerator Division, Thomas Jefferson National Accelerator Facility, Newport News, Virginia 23606, USA
}

H. Wang

Accelerator Division, Thomas Jefferson National Accelerator Facility, Newport News, Virginia 23606, USA

(Received 23 March 2009; published 18 June 2009)

\begin{abstract}
A new type of rf structure for the deflection and crabbing of particle beams is presented. The structure is comprised of a number of parallel TEM resonant lines operating in opposing phase from each other. One of its advantages is its compactness compared to conventional crabbing cavities operating in the $\mathrm{TM}_{110}$ mode, thus allowing low frequency designs. This geometry would also be effective for the deflection of beams propagating at velocities substantially less than that of light.
\end{abstract}

DOI: 10.1103/PhysRevSTAB.12.062002

PACS numbers: 29.27.Ac, 41.85.Ar, 41.85.Ct

\section{INTRODUCTION}

Rf cavities for the deflection or crabbing of particle beams have been developed for many years. Conceptually, deflecting and crabbing structures are identical; the former operate in the mode where the center of the bunch experiences a finite (often maximum) integrated Lorentz transverse force while the latter operate in the mode where the center of the bunch experiences a zero integrated Lorentz force but the front and tail of the bunch are deflected in opposite directions. The majority of deflecting/crabbing structures are single-cell or multicell superconducting cavities operating in the $\mathrm{TM}_{110}$ mode [1-4], although some are room temperature structures operating in a $\lambda / 4$ mode [5] or are of $H$ type [6]. Deflecting cavities have been used to deliver a particle beam, often simultaneously, to a number of experimental stations [7], while crabbing rf structures have been of interest for the increase of luminosity in colliders - both circular and linear [8,9] — and, more recently, for the generation of subpicosecond $\mathrm{x}$-ray pulses [10,11].

The concept of the parallel-bar deflecting structure is shown in Fig. 1; it consists of two parallel $\lambda / 2$ TEM resonant lines with the beam line perpendicular to the bars and passing between them. This structure has two fundamental TEM modes. In the deflecting mode, referred to as the $\pi$ mode, the two parallel bars oscillate in opposite phase. The voltages generated are maximum and of opposite sign in the middle of the bars and generate a transverse electric field as shown in Fig. 2. The magnetic field is null in the midplane containing the beam line and is maximum where the bars meet the shorting planes, as shown in Fig. 3. Thus, unlike $\mathrm{TM}_{110}$ structures where the deflection is produced by interaction with the magnetic field, in the

\footnotetext{
*delayen@jlab.org
}

parallel-bar structure the deflection is produced by interaction with the electric field.

In the other fundamental mode, referred to as the 0 mode, the bars oscillate in phase. The electric field profile in the midplane and the magnetic field profile in the top or bottom plane are shown in Figs. 4 and 5 respectively. The 0 mode could be used as an accelerating mode, although it is much better implemented in the coaxial half-wave geometry where the two bars have been merged into one [12].

\section{ANALYTICAL MODEL}

If the distance between the side walls and the resonant bars is substantially larger than the distance between the bars and the vertical symmetry plane, then the walls' contributions to the electromagnetic properties will be

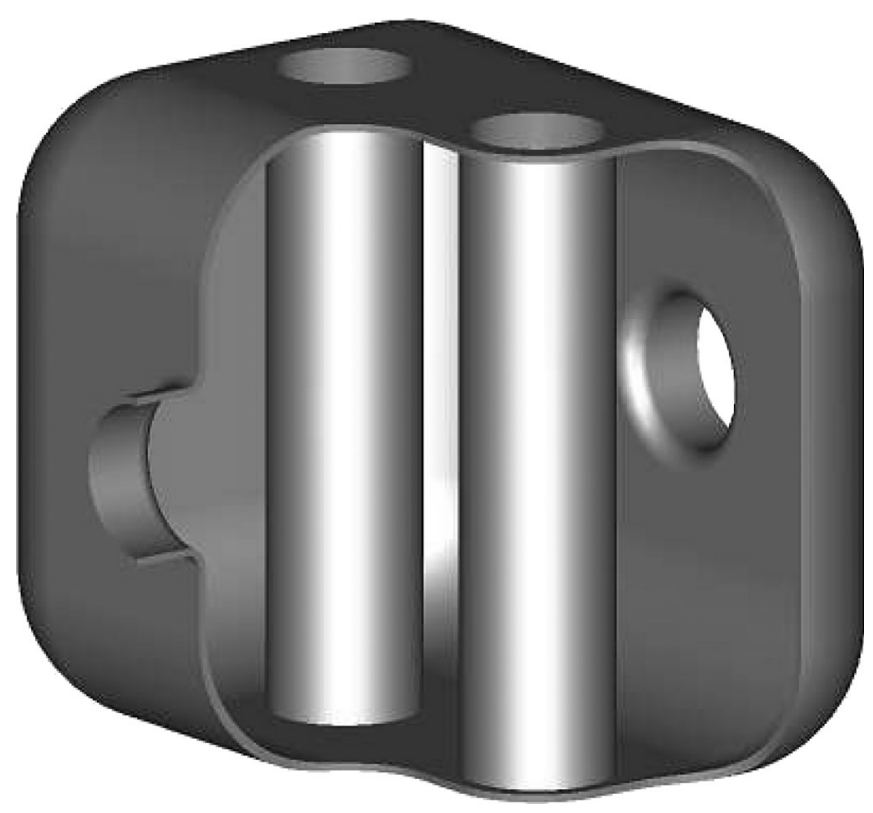

FIG. 1. Concept of a single-cell parallel-bar rf structure. 


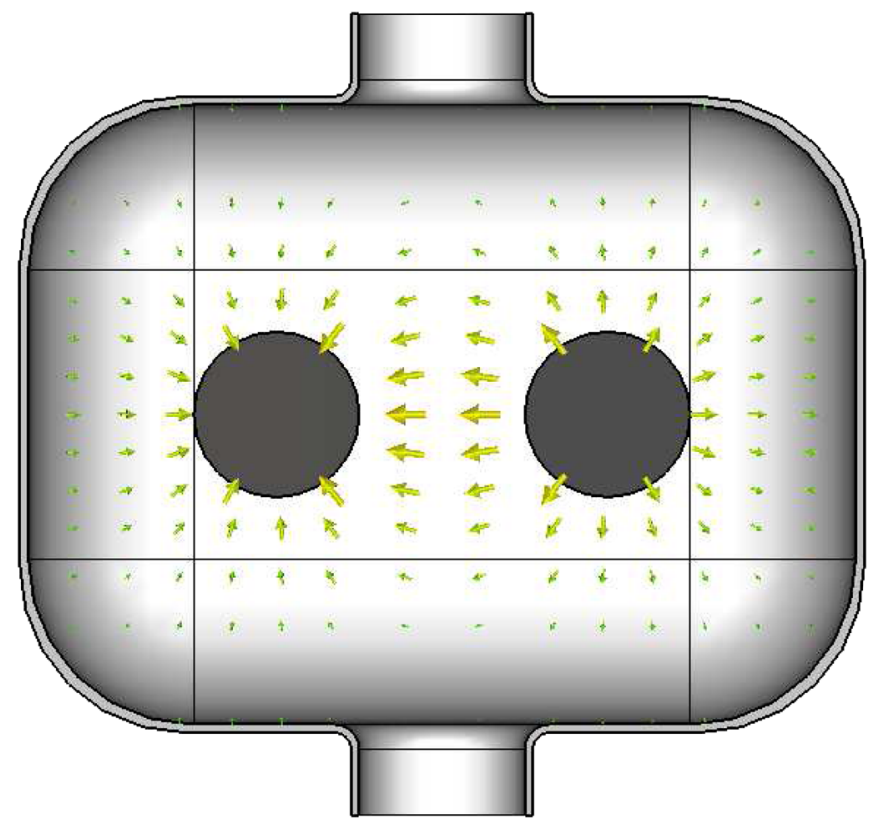

FIG. 2. (Color) Electric field in the midplane of the parallel-bar structure operating in the $\pi$ mode.

small and the fundamental cell can be modeled by two parallel infinite planes separated by $\lambda / 2$ and joined by two parallel cylinders of radius $R$ and of axis-to-axis separation $2 A$. The properties of such a structure can be calculated exactly as shown in the Appendix. They are summarized below and will be compared to simulations of actual structures later.

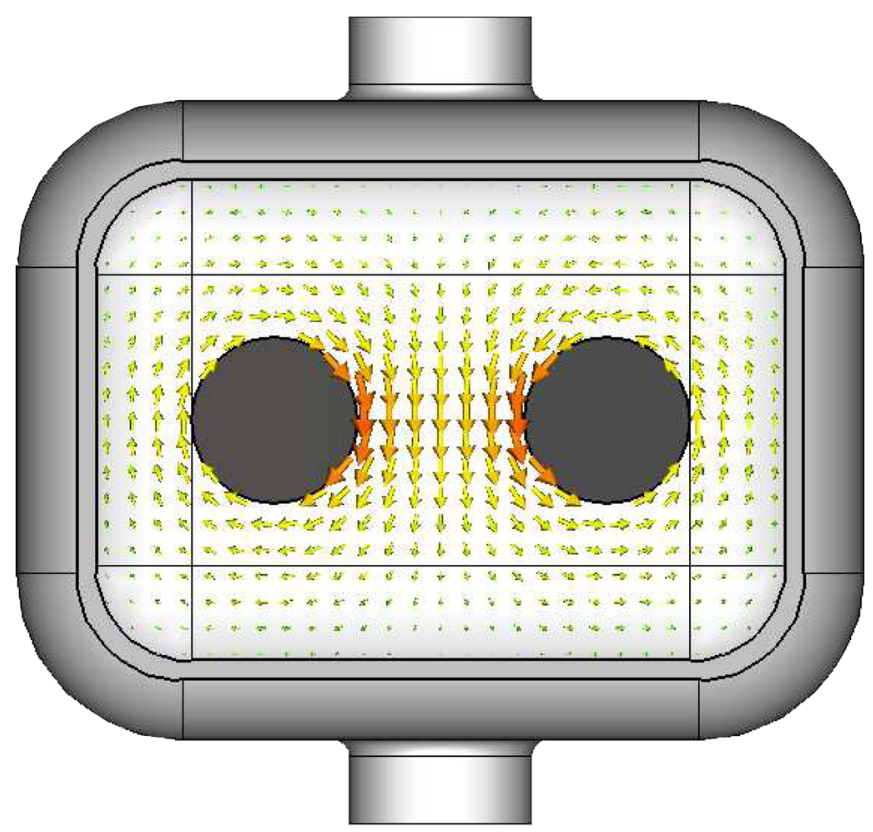

FIG. 3. (Color) Magnetic field in the top plate of the parallel-bar structure operating in the $\pi$ mode.

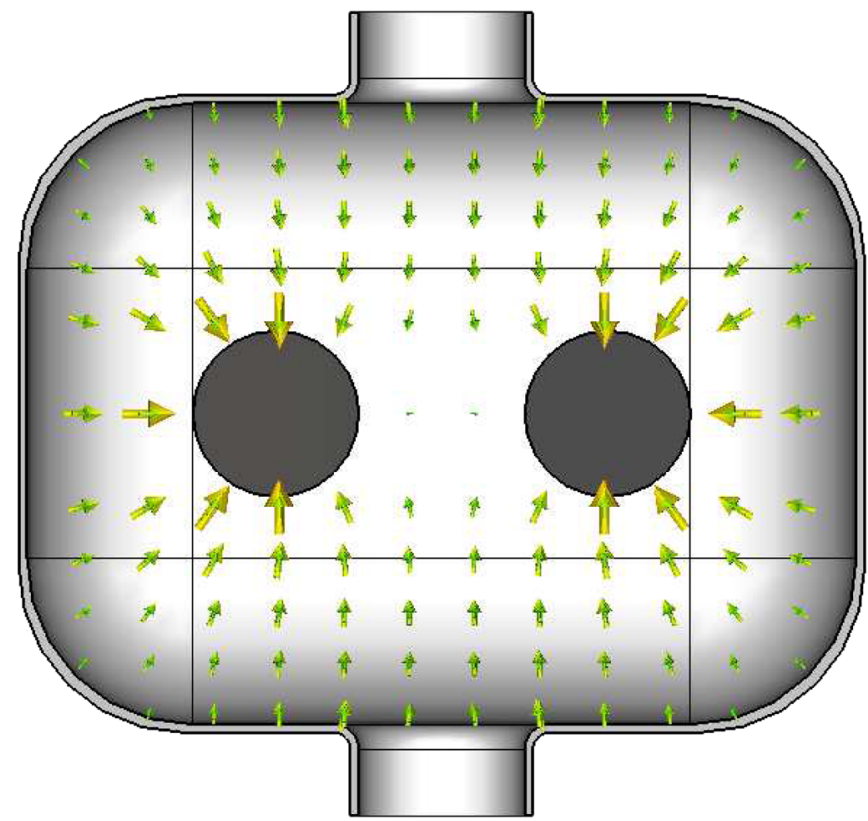

FIG. 4. (Color) Electric field in the midplane of the parallel-bar structure operating in the 0 mode.

It can be noted that the electromagnetic properties can be expressed simply as functions of $R / \lambda$ and $\alpha=A / R$, where $\lambda$ is the free-space wavelength corresponding to the frequency of the fundamental TEM mode.

\section{A. Peak surface electric field}

For a velocity-of-light particle, the length of the cavity along the beam line would be close to $\lambda / 2$ and it is natural

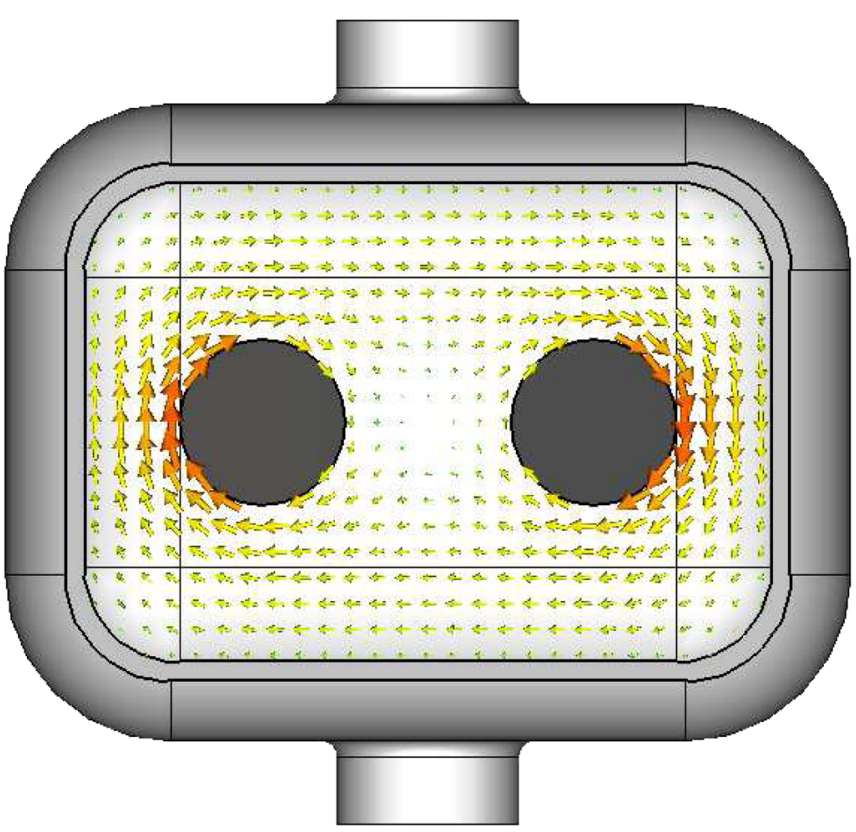

FIG. 5. (Color) Magnetic field in the top plate of the parallel-bar structure operating in the 0 mode. 


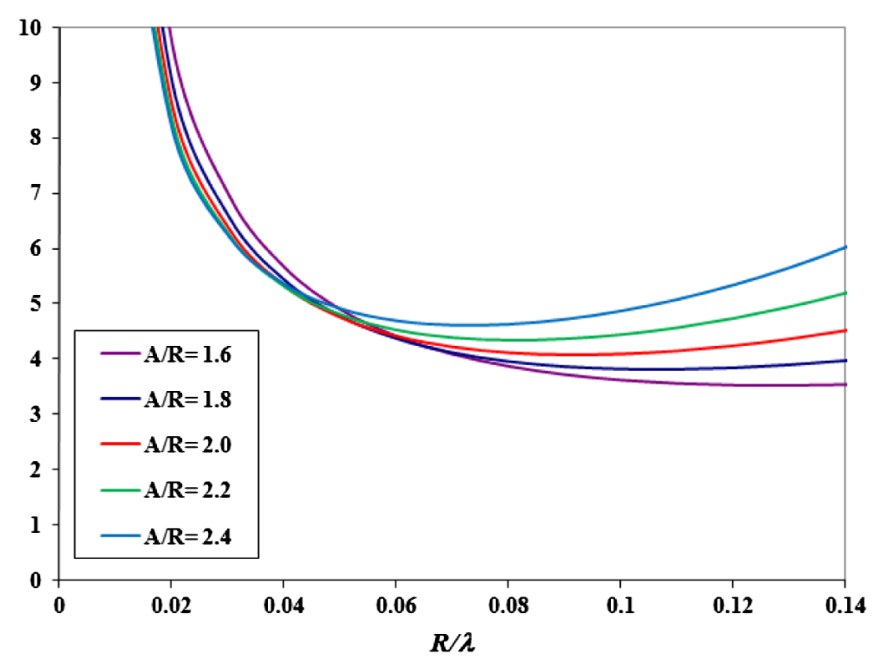

FIG. 6. (Color) $E_{p} / E_{t}$, ratio of peak-to-transverse electric field given by Eq. (1). $R$ is the radius of the bars and $2 A$ is the distance between their axes.

to define the transverse deflecting electric field as $E_{t}=$ $2 V_{t} / \lambda$, where $V_{t}$ is the transverse voltage acquired by an on-crest particle. The ratio of peak surface electric field and transverse deflecting field is given by

$$
\frac{E_{p}}{E_{t}}=\frac{1}{4 \pi} \frac{\lambda}{R}\left(\frac{\alpha+1}{\alpha-1}\right)^{1 / 2} \exp \left[2 \pi \frac{R}{\lambda} \sqrt{\alpha^{2}-1}\right]
$$

and shown in Fig. 6.

\section{B. Peak surface magnetic field}

Since, in this model, the electromagnetic mode is purely TEM, the magnetic field at any location is proportional to the electric field at the location with the same transverse coordinates but separated by $\lambda / 4$ in the direction parallel to the bars' axes [see Eqs. (A1) and (A2)]. Thus, the peak surface magnetic field is related to the peak surface electric

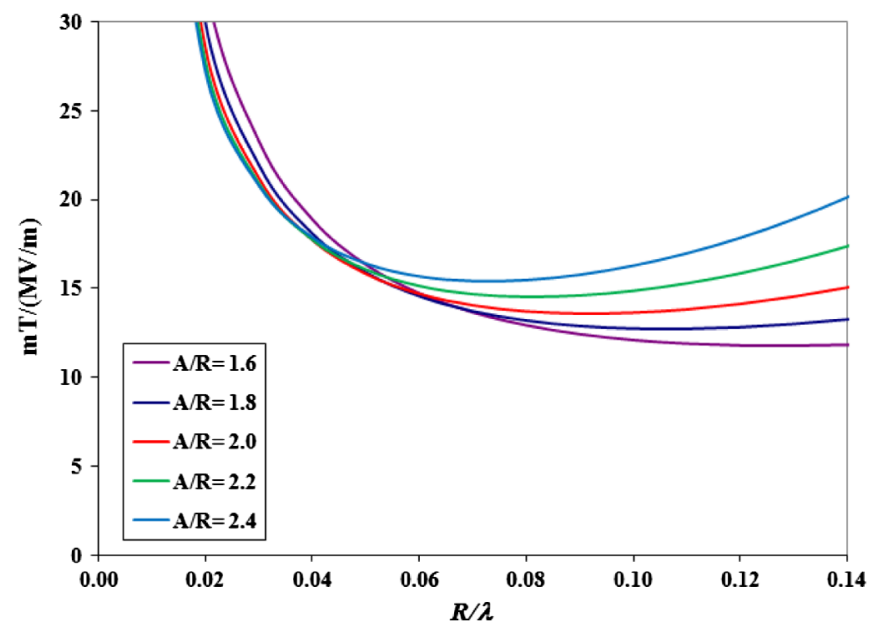

FIG. 7. (Color) $B_{p} / E_{t}$, ratio of peak surface magnetic field to transverse electric field given by Eq. (2). field by

$$
B_{p}(\text { in } \mathrm{mT})=\frac{10^{9}}{c} E_{p}(\text { in } \mathrm{MV} / \mathrm{m}) .
$$

The ratio of the peak magnetic field and the transverse deflecting field is shown in Fig. 7.

\section{Energy content}

The energy content $U$ is related to the transverse gradient $E_{t}$ by

$$
U=E_{t}^{2} \frac{\epsilon_{0}}{32 \pi} \lambda^{3} \cosh ^{-1}(\alpha) \exp \left[4 \pi \frac{R}{\lambda} \sqrt{\alpha^{2}-1}\right],
$$

where $\epsilon_{0}$ is the permittivity of vacuum. The energy content at $E_{t}=1 \mathrm{MV} / \mathrm{m}$ and a wavelength of $1 \mathrm{~m}$ is shown in Fig. 8.

\section{Geometrical factor}

The geometrical factor $G$-the product of the quality factor $Q$ and the surface resistance $R_{s}$-is given by

$$
G=Q R_{s}=2 \pi Z_{0} \frac{R}{\lambda} \frac{\cosh ^{-1}(\alpha)}{\frac{8 R}{\lambda} \cosh ^{-1}(\alpha)+\frac{\alpha}{\sqrt{\alpha^{2}-1}}},
$$

where $Z_{0}=\sqrt{\mu_{0} / \epsilon_{0}} \approx 377 \Omega$ is the impedance of the vacuum. A plot of the geometrical factor is shown in Fig. 9.

\section{E. Transverse $R / Q$}

Defining the transverse shunt impedance $R_{t}=V_{t}^{2} / P$, where $P$ is the power dissipation, $R_{t} / Q$ is given by

$$
R_{t} / Q=4 Z_{0} \frac{\exp \left[-4 \pi \frac{R}{\lambda} \sqrt{\alpha^{2}-1}\right]}{\cosh ^{-1}(\alpha)},
$$

and shown in Fig. 10.

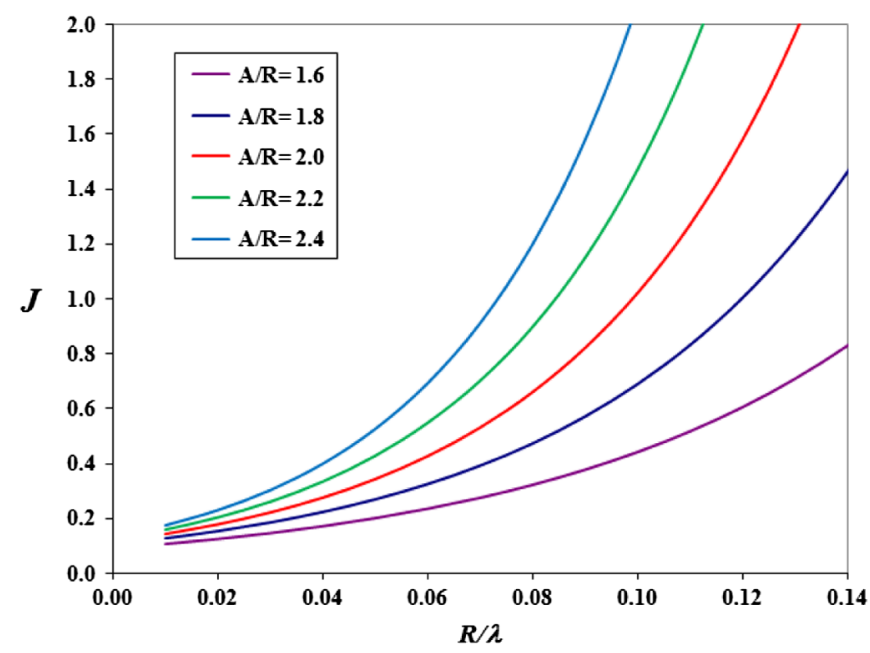

FIG. 8. (Color) $U /\left(E_{t}^{2} / \lambda^{2}\right)$, energy content given by Eq. (3) at a transverse gradient of $1 \mathrm{MV} / \mathrm{m}$ for a cavity of wavelength of $1 \mathrm{~m}$. 


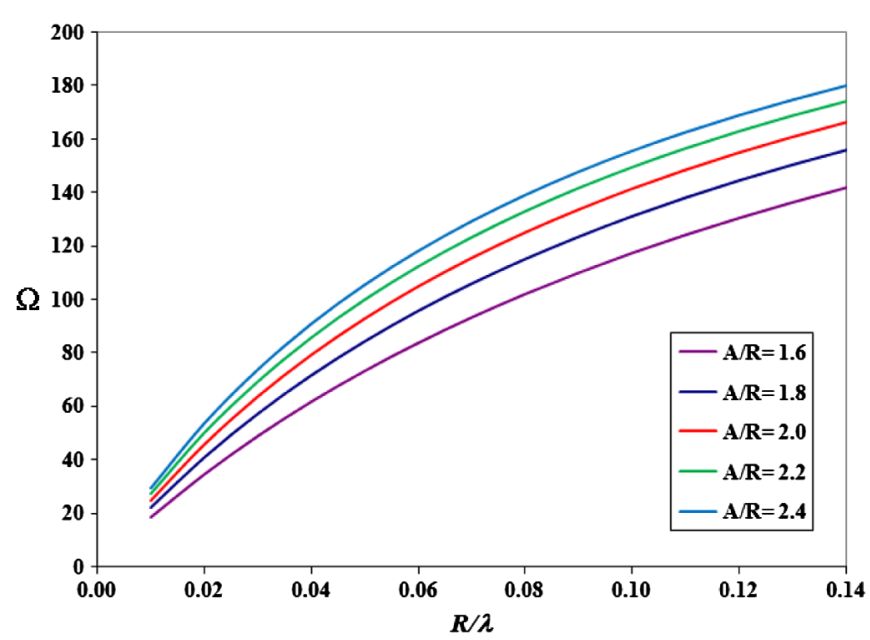

FIG. 9. (Color) $G=Q R_{s}$, geometrical factor given by Eq. (4).

\section{F. Transverse shunt impedance}

The product of the transverse shunt impedance $R_{t}=$ $V_{t}^{2} / P$ and the surface resistance $R_{s}$ of the structure material is given by

$$
R_{t} R_{s}=8 \pi Z_{0}^{2} \frac{R}{\lambda} \frac{\exp \left[-4 \pi \frac{R}{\lambda} \sqrt{\alpha^{2}-1}\right]}{\frac{8 R}{\lambda} \cosh ^{-1}(\alpha)+\frac{\alpha}{\sqrt{\alpha^{2}-1}}},
$$

and is shown in Fig. 11.

It can be seen from Figs. 6 and 7 that the peak surface electric and magnetic fields depend weakly on $A / R$ and have a broad minimum for rather large values of $R / \lambda$. On the other hand, the transverse shunt impedance has a much stronger dependence on $A / R$ and is maximum for smaller $R / \lambda$. Thus, the final design would depend on which parameter to optimize and, in particular, whether the structure will be room temperature or superconducting.

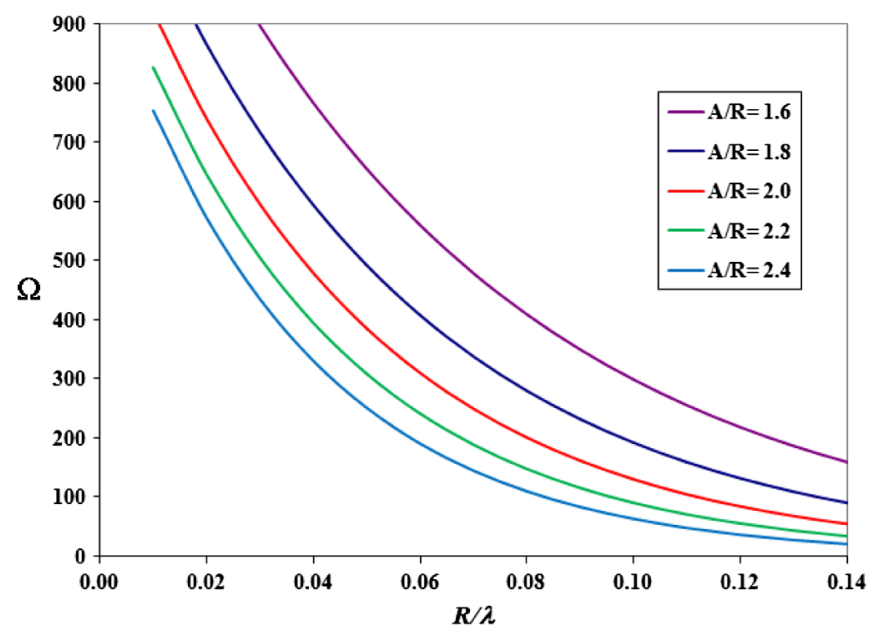

FIG. 10. (Color) $R_{t} / Q$, ratio of transverse shunt impedance and quality factor given by Eq. (5).

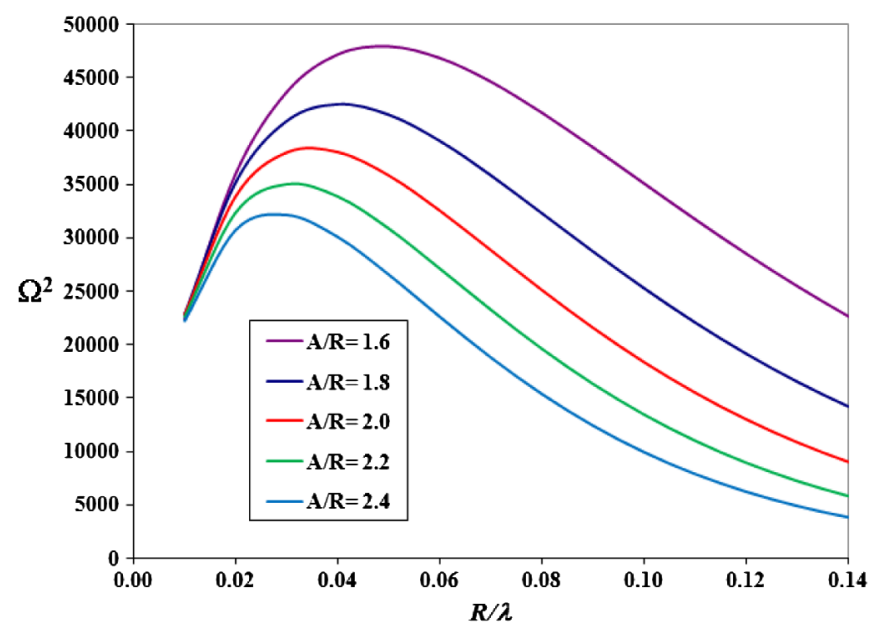

FIG. 11. (Color) $R_{t} R_{s}=G \times R_{t} / Q$, product of the transverse shunt impedance and the surface resistance given by Eq. (6).

\section{ELECTROMAGNETIC DESIGN}

Since one of the main features of this geometry is its small transverse size, it would be particularly attractive at low frequency. One possible application would be a $500 \mathrm{MHz}$ deflector for the Jefferson Lab $12 \mathrm{GeV}$ upgrade [13]. This structure might also be an option for a crabbing cavity for the LHC upgrade [14-16]. The properties of a $400 \mathrm{MHz}$ single-cell cavity, shown in Fig. 1, obtained from simulations using CST MICROWAVE STUDIO ${ }^{\circledR}$ are compared to the analytical results of the previous section in Fig. 12. The lengths of the bars and that of the outer box along the beam line were, to first order, fixed at $375 \mathrm{~mm}$ and the main design parameters were the radii and the separation of the parallel bars. The electromagnetic properties of the structure are relatively insensitive to the width of the box (in the direction perpendicular to the beam line) as long as those walls are sufficiently far from the bars. Each marker on the left-hand side plots represents a structure geometry that was analyzed. The electromagnetic simulations compare very favorably to the analytical results.

As was expected, the transverse shunt impedance of this design is quite high compared to designs based on $\mathrm{TM}_{110}$ modes. This is similar to the high shunt impedance of TEM accelerating structures compared to $\mathrm{TM}_{010}$ structures [12].

For velocity-of-light applications, TEM accelerating structures have peak surface fields larger than $\mathrm{TM}_{010}$ structures [12]. The analytical model and these simulations show that this is not the case for deflecting cavities as peak surface fields for TEM structures are comparable to those of $\mathrm{TM}_{110}$ structures.

In the deflecting $\pi$ mode there is no longitudinal electric field on the beam axis while there is a longitudinal electric field in the accelerating 0 mode; neither mode has a magnetic field on the beam axis. Thus, the presence of the beam line apertures will have little effect on the $\pi$-mode frequency while raising the frequency of the 0 mode through 

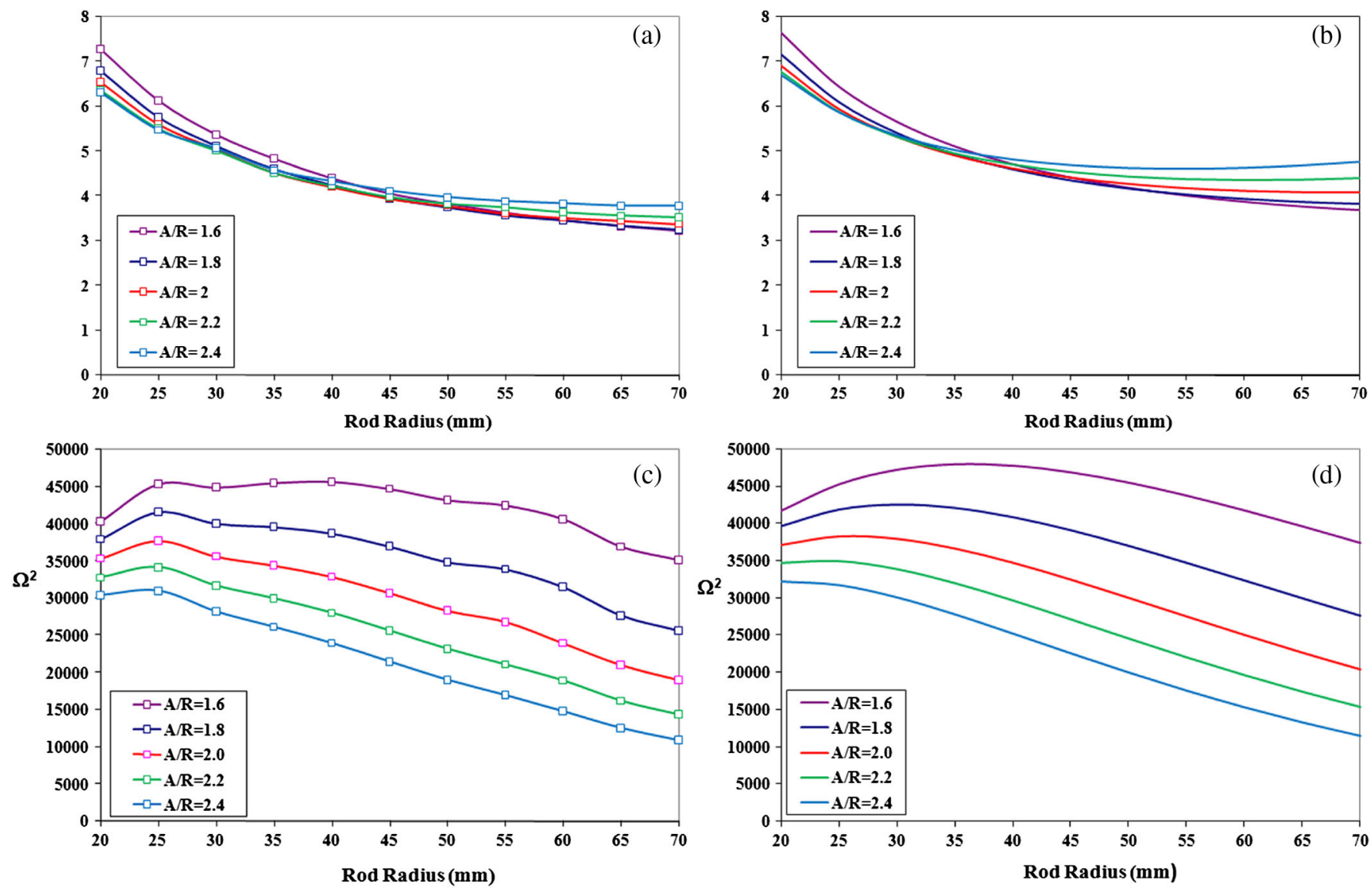

FIG. 12. (Color) Comparison between results of electromagnetic code simulation using MICROWAVE STUDIO (left) of a $400 \mathrm{MHz}$ geometry shown in Fig. 1, and analytical results of Sec. II (right). The upper plots are for $E_{p} / E_{t}$, the ratio of peak-to-transverse electric field, and the lower plots are for $R_{t} R_{s}$, the transverse shunt impedance. Each marker on the left-hand plots represents a structure geometry that was analyzed.

TABLE I. Properties of the parallel-bar structure shown in Fig. 1 calculated from OMEGA3P and the analytical model of Fig. 18.

\begin{tabular}{lccc}
\hline \hline Parameter & OMEGA3P & Analytical model & Unit \\
\hline Frequency of $\pi$ mode & 400 & 400 & $\mathrm{MHz}$ \\
$\lambda / 2$ of $\pi$ mode & 374.7 & 374.7 & $\mathrm{~mm}$ \\
Frequency of 0 mode & 414.3 & 400 & $\mathrm{MHz}$ \\
Cavity length & 374.7 & $\infty$ & $\mathrm{mm}$ \\
Cavity width & 500 & $\infty$ & $\mathrm{mm}$ \\
Bars length & 385.0 & 374.7 & $\mathrm{~mm}$ \\
Bars diameter $(2 R)$ & 100 & 100 & $\mathrm{~mm}$ \\
Bars axes separation $(2 A)$ & 200 & 200 & $\mathrm{~mm}$ \\
Aperture diameter & 100 & 0 & $\mathrm{~mm}$ \\
Deflecting voltage $V_{t}^{\mathrm{a}}$ & 0.375 & 0.375 & $\mathrm{MV}$ \\
Peak electric field $E_{p}{ }^{\mathrm{a}}$ & 4.09 & 4.28 & $\mathrm{MV} / \mathrm{m}$ \\
Peak magnetic field $B_{p}{ }^{\mathrm{a}}$ & 13.31 & 14.24 & $\mathrm{mT}$ \\
Energy content $U^{\mathrm{a}}$ & 0.215 & 0.209 & $\mathrm{~J}$ \\
Geometrical factor $G=Q R_{s}$ & 96.0 & 112 & $\Omega$ \\
$R_{t} / Q$ & 260 & 268 & $\Omega$ \\
$R_{t} R_{s}$ & $2.50 \times 10^{4}$ & $3.00 \times 10^{4}$ & $\Omega^{2}$ \\
\hline \hline
\end{tabular}

${ }^{\mathrm{a}}$ At $E_{t}=1 \mathrm{MV} / \mathrm{m}$. 
the work done by the radiation pressure. This is a direct consequence of the Boltzmann-Ehrenfest adiabatic theorem which in its microwave incarnation is known as Slater's formula [17]. Near the edges and corners at the top and bottom plates both modes will have a magnetic field but the field is higher in the 0 mode than in the $\pi$ mode. Thus, the rounding of the corners will raise the frequency of the 0 mode by a larger amount than that of the $\pi$ mode. The beam line apertures and the rounding of the edges and corners will contribute to the removal of the degeneracy by raising the frequency of the 0 mode above that of the $\pi$ mode.

Properties of a preliminary design of a $400 \mathrm{MHz}$ parallel-bar deflecting structure obtained from OMEGA3P [18] are shown in Table I. It can be noted that, as was expected from the above discussion, the deflecting $\pi$ mode is the lowest frequency mode; this would simplify the damping of all the other modes in high current applications.

The parallel-bar structure discussed so far can be straightforwardly extended to a multicell structure by the addition of sets of parallel bars, as shown in Fig. 13 for a two-cell structure. The spacing between successive pairs of bars would be $\beta \lambda / 2$, where $\beta c$ is the velocity of the particle. In such a multicell structure the number of nearly degenerate TEM modes is equal to the number of bars, and the deflecting mode of interest is the $(\pi, \pi)$ mode where the bars in each pair are in opposite phase with each other, and the bars in adjacent pairs also oscillate in opposite phase. Perturbation of the outer geometry (such as rounding of the corners or wall deformation at appropriate locations) is necessary to provide the required splitting between the $(\pi, \pi)$ mode and all the others. As long as the number of cells is reasonably small, the deflecting $(\pi, \pi)$ mode will have the lowest frequency. As the cavity length is increased, the frequency of one of the resonant modes of the outer enclosure would become lower than that

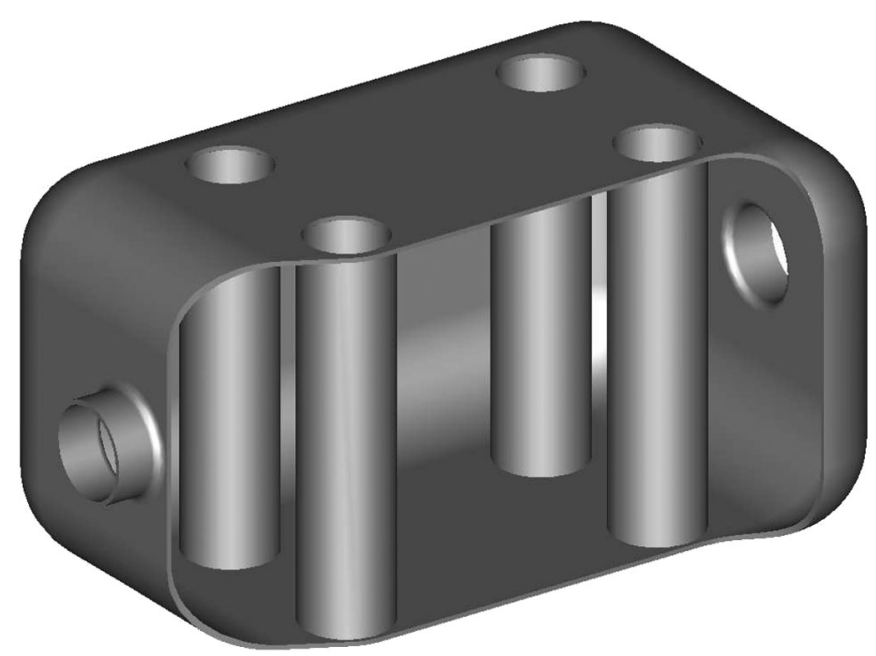

FIG. 13. Concept of a two-cell parallel-bar rf structure.

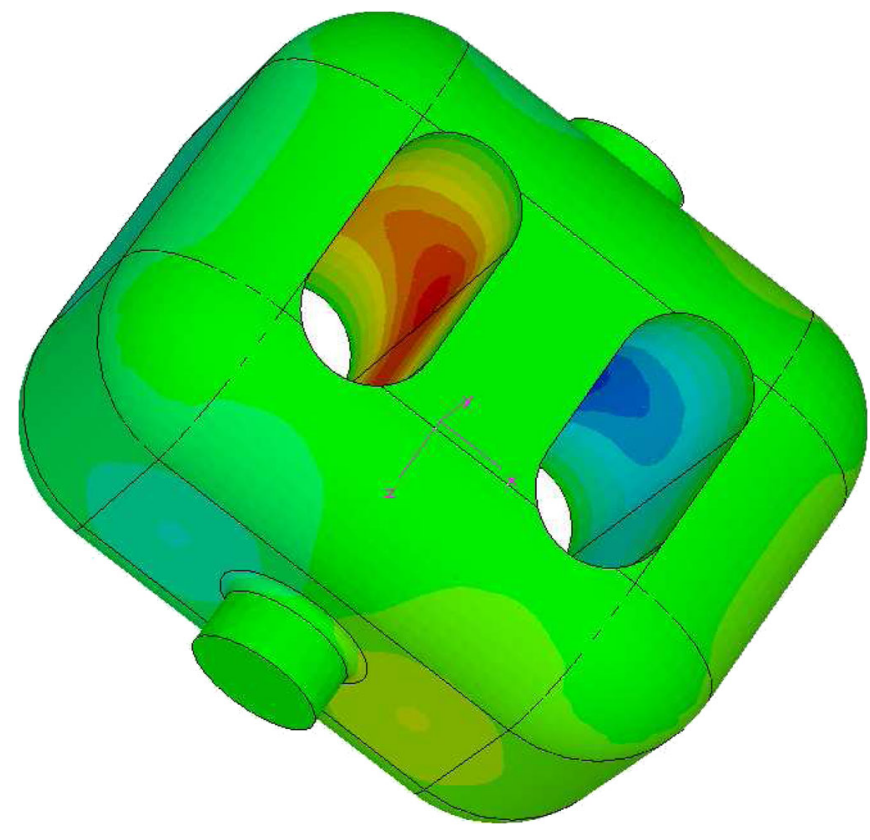

FIG. 14. (Color) Surface electric field in a parallel-bar structure with rounded rectangular bars operating in the $\pi$ mode.

of the $(\pi, \pi)$ mode which, in certain applications, would be undesirable.

All the previous geometries discussed so far use straight circular cylinders of constant cross section for the bars. The performance of the parallel-bar geometry can be further improved by deviations from that basic geometry. Figures 14 and 15 show a parallel-bar structure where the

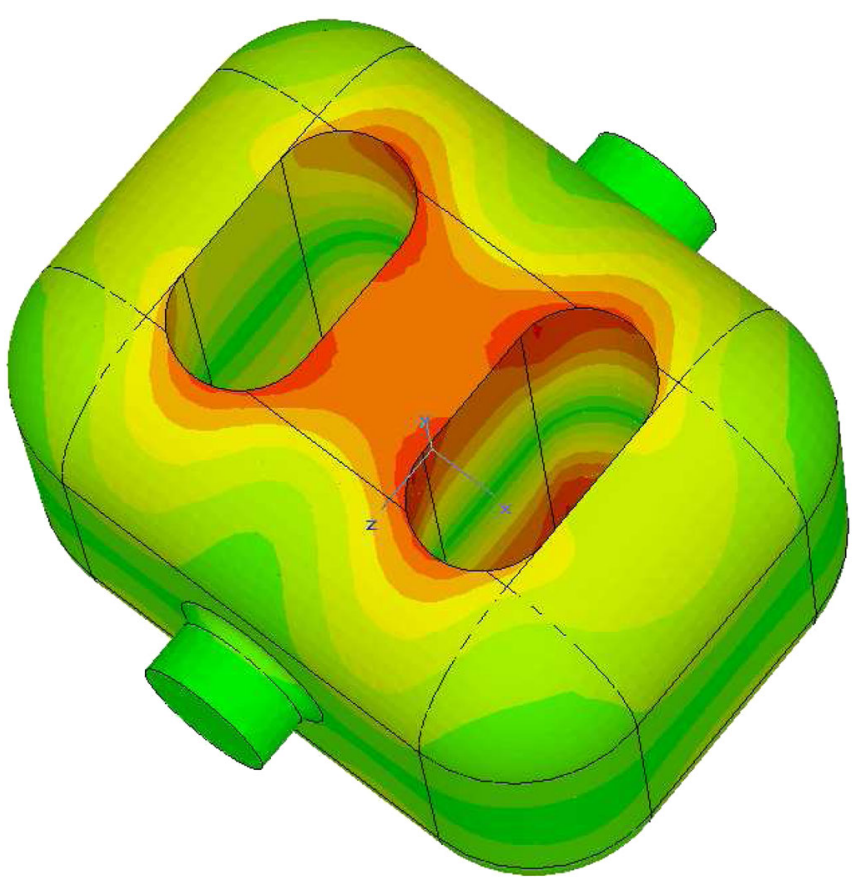

FIG. 15. (Color) Surface magnetic field in a parallel-bar structure with rounded rectangular bars operating in the $\pi$ mode. 
bars have a rounded rectangular cross section. In TEM accelerating structures-such as the coaxial-half-wave or the spoke resonator - the voltage acquired by the particle is essentially dictated by the voltage generated on the center conductor. The goal of the center conductor design is then to minimize the surface electric and magnetic fields for a given voltage. In a deflecting structure, the transverse voltage acquired by the particle depends not only on the magnitude of the transverse field generated by the voltage between the bars but also on its extent, i.e., the amount of time the particle will interact with the transverse field. Thus, in this case, it is worth exploring cross-section geometries that, for a given voltage on the bars, could have larger surface fields than a circular cross section as long as the transverse field extends over a larger distance. The rounded rectangular geometry shown in Figs. 14 and 15 might have a larger surface field at a given voltage on the bars than a circular cross section, but it would also generate a transverse field over a larger extent of the particle trajectory and thus have a lower surface field at a given deflecting voltage. This is illustrated in Table II where the properties of two similar parallel-bar resonator, one with a circular cross section, the other with a rounded rectangular cross section, are compared. It is apparent that that simple modification of the cross section leads to a substantial reduction of the peak surface fields while increasing the transverse shunt impedance.

For a superconducting cavity, a likely limitation will be the surface magnetic field which is maximum where the bars meet the top and bottom plates. A standard feature in TEM accelerating cavities is to flare the center conductor into an hourglass or hyperboloidal shape; this increases the

TABLE II. Properties of the parallel-bar structures shown in Figs. 1 and 14 calculated from OMEGA3P.

\begin{tabular}{lccc}
\hline \hline Parameter & Figure 1 & Figure 14 & Unit \\
\hline Frequency of $\pi$ mode & 400 & 400 & $\mathrm{MHz}$ \\
$\lambda / 2$ of $\pi$ mode & 374.7 & 374.7 & $\mathrm{~mm}$ \\
Frequency of 0 mode & 414.4 & 419.6 & $\mathrm{MHz}$ \\
Cavity length & 374.7 & 374.7 & $\mathrm{~mm}$ \\
Cavity width & 500 & 500 & $\mathrm{~mm}$ \\
Bars length & 385.0 & 392.2 & $\mathrm{~mm}$ \\
Bars thickness $(2 R)$ & 100 & 100 & $\mathrm{~mm}$ \\
Bars width & 100 & 200 & $\mathrm{~mm}$ \\
Bars axes separation $(2 A)$ & 200 & 200 & $\mathrm{~mm}$ \\
Aperture diameter & 100 & 100 & $\mathrm{~mm}$ \\
Deflecting voltage $V_{t}^{\mathrm{a}}$ & 0.375 & 0.375 & $\mathrm{MV}$ \\
Peak electric field $E_{p}{ }^{\mathrm{a}}$ & 4.09 & 2.69 & $\mathrm{MV} / \mathrm{m}$ \\
Peak magnetic field $B_{p}{ }^{\mathrm{a}}$ & 13.31 & 8.17 & $\mathrm{mT}$ \\
Energy content $U^{\mathrm{a}}$ & 0.215 & 0.160 & $\mathrm{~J}$ \\
Geometrical factor $G=Q R_{s}$ & 96.0 & 91.3 & $\Omega$ \\
$R_{t} / Q$ & 260 & 350 & $\Omega$ \\
$R_{t} R_{s}$ & $2.50 \times 10^{4}$ & $3.20 \times 10^{4}$ & $\Omega^{2}$ \\
\hline \hline
\end{tabular}

${ }^{\mathrm{a}}$ At $E_{t}=1 \mathrm{MV} / \mathrm{m}$.

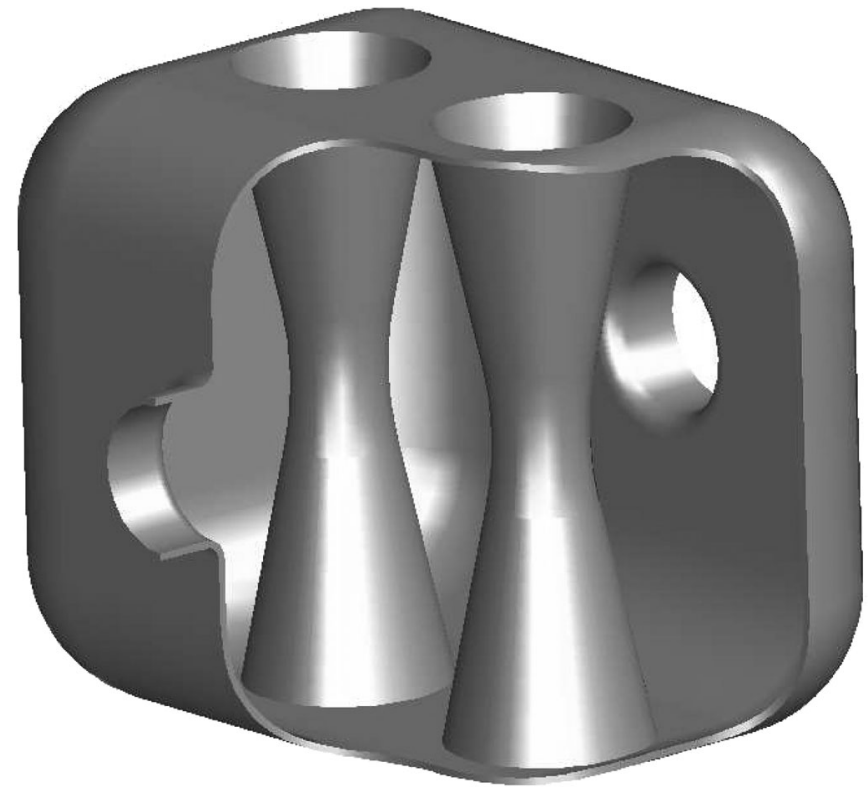

FIG. 16. Concept of a TEM deflecting structure with parallel bars of variable cross section.

cross section of the bars in the region of maximum magnetic field, resulting in its reduction. Such a feature can also be implemented in the parallel-bar cavity as shown in Fig. 16. This feature is particularly effective in accelerating multispoke cavities where adjacent resonant lines are perpendicular to each other. In a parallel-bar structure this flaring can only be done to a limited extent since, at some point, the bars will become too close, thus "crowding" magnetic field, leading to an increase of the magnetic field.

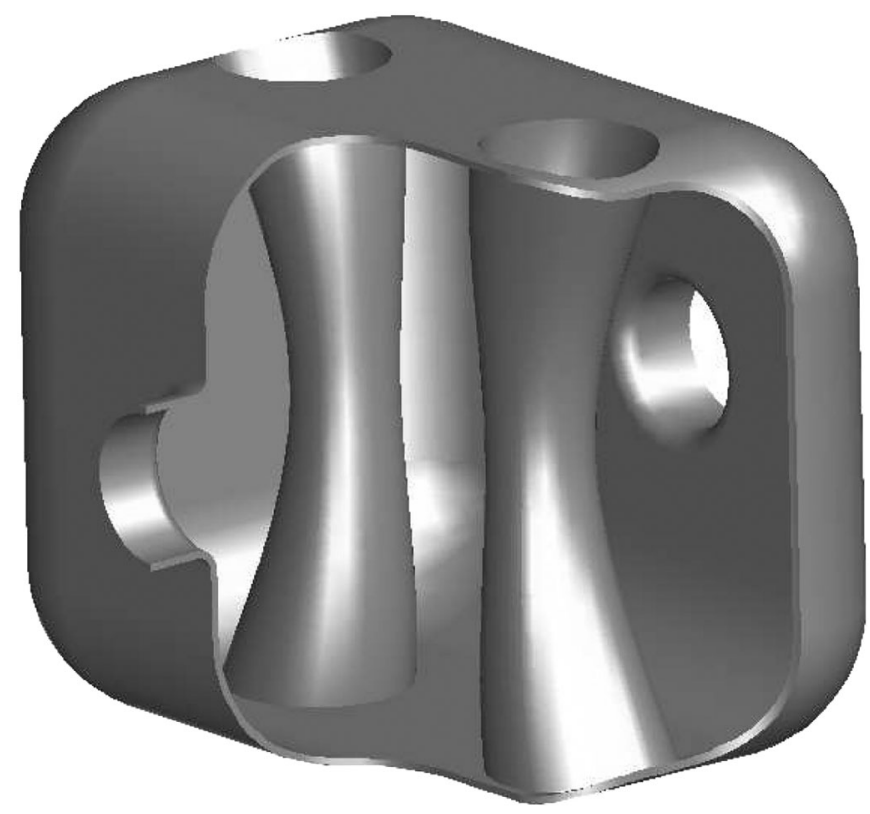

FIG. 17. Concept of a TEM deflecting structure with curved resonant bars. 
One way to increase the cross section while avoiding that crowding is to use curved resonant bars as shown in Fig. 17. The bars would be closest at their center but bending away from each other as they near the top and bottom planes.

All these modifications from the basic geometry can be implemented simultaneously, leading to an expected improvement in performance at the expense of an increased degree of engineering complexity.

While most of the above analysis and discussion referred to beams propagating at the velocity of light, this geometry would also be effective for the deflection of beams propagating at velocities much less than that of light. This can be easily accomplished by scaling all the dimensions parallel to the beam line by $\beta=v / c$ while keeping unchanged all the dimensions perpendicular to the beam line.

\section{SUMMARY}

A new type of deflecting/crabbing structure has been presented and analyzed. It is based on TEM resonant lines and provides deflection through interaction with the electric field. This new geometry is much smaller than a $\mathrm{TM}_{110}$ cavity of the same frequency. It also offers a high shunt impedance and low surface fields. As in all the other TEMtype cavities, the couplers for the fundamental and higherorder modes could be located on the outer walls instead of on the beam line since the surface fields on the outer enclosure are small. This feature makes better use of beam line space while easing the extraction of higher-order modes and preventing the possibility of trapped modes.

\section{ACKNOWLEDGMENTS}

This manuscript has been authored by Jefferson Science Associates, LLC under U.S. DOE Contract No. DE-AC0506OR23177. The U.S. Government retains a nonexclusive, paid-up, irrevocable, worldwide license to publish or reproduce this manuscript for U.S. Government purposes.

\section{APPENDIX: DERIVATION OF ELECTROMAGNETIC PROPERTIES OF ANALYTICAL MODEL}

In the simple model shown in Fig. 18, the rf deflecting structure is represented by two infinite parallel planes separated by $\lambda / 2$ and joined by two cylinders perpendicular to the planes, of radius $R$ and axis-to-axis distance $2 A$. Since the geometry has translational invariance and is multiply connected, it will support pure transverse electromagnetic (TEM) modes of frequency $\omega=2 \pi c / \lambda$. The number of such modes will be equal to the number of bars. In one of the modes - the $\pi$ mode-the two bars resonate in opposite phase, while in the other-the 0 mode-they resonate in phase. The $\pi$ mode is the deflecting mode while the 0 mode is an accelerating mode. In such modes, the electric field in each plane satisfies Poisson's
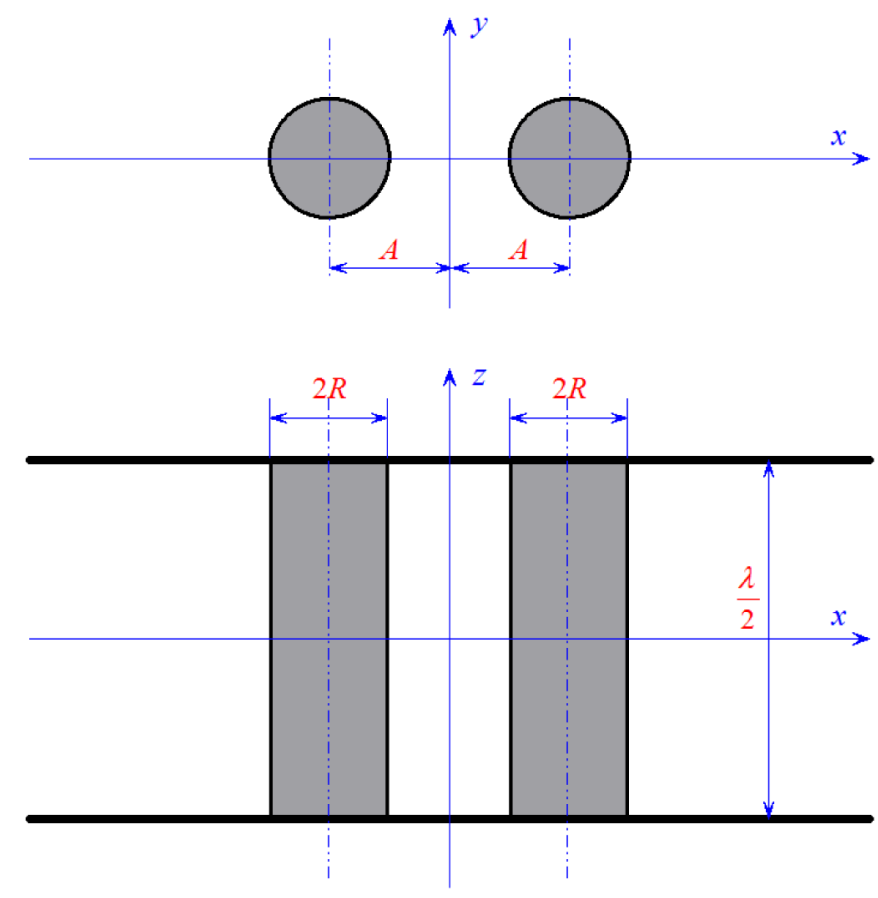

FIG. 18. (Color) Model of a one-cell parallel-bar rf structure.

equation and is the electrostatic problem of two infinitely long equipotential cylinders. For the $\pi$ mode this can be further simplified to two infinite parallel lines of uniform and opposite linear charge since, in this case, the equipotential are cylinders. Once the electrostatic problem has been solved and the planar electric field $\vec{E}_{t}(x, y)$ is obtained, the electric and magnetic fields in a TEM resonant structure are

$$
\begin{gathered}
\vec{E}_{t}(x, y, z, t)=\vec{E}_{t}(x, y) \cos \left(\frac{2 \pi z}{\lambda}\right) \cos (\omega t), \\
\vec{H}_{t}(x, y, z, t)=\hat{z} \times \frac{\vec{E}_{t}(x, y)}{Z_{0}} \sin \left(\frac{2 \pi z}{\lambda}\right) \sin (\omega t) .
\end{gathered}
$$

Assuming two infinitely long lines, parallel to the $z$ axis, and crossing the $(x, y)$ plane at $x= \pm a, y=0$, and carrying uniform linear charge per unit length $\mp q$, the potential is given by

$$
V(x, y)=\frac{q}{4 \pi \epsilon_{0}} \ln \left(\frac{r_{-}^{2}}{r_{+}^{2}}\right)
$$

with

$$
\begin{aligned}
& r_{-}^{2}=(x-a)^{2}+y^{2}, \\
& r_{+}^{2}=(x+a)^{2}+y^{2} ;
\end{aligned}
$$

and the electric field is

$$
E_{x}(x, y)=-\frac{\partial V}{\partial x}=-\frac{a q}{\pi \epsilon_{0}}\left[\frac{x^{2}-a^{2}-y^{2}}{r_{-}^{2} r_{+}^{2}}\right],
$$




$$
E_{y}(x, y)=-\frac{\partial V}{\partial y}=-\frac{2 a q}{\pi \epsilon_{0}}\left[\frac{x y}{r_{-}^{2} r_{+}^{2}}\right] .
$$

The equipotential surface $V(x, y)=V_{0}$ is given by

$$
\frac{r_{-}^{2}}{r_{+}^{2}}=\frac{(x-a)^{2}+y^{2}}{(x+a)^{2}+y^{2}}=e^{2 \mu}
$$

where

$$
\mu=\frac{2 \pi \epsilon_{0} V_{0}}{q} .
$$

This implies

$$
(x+a \operatorname{coth} \mu)^{2}+y^{2}=\left(\frac{a}{\sinh \mu}\right)^{2} .
$$

Thus two infinite parallel cylinders, at potentials $\pm V_{0}$, of radius $R$ and axis separation $2 A$, can be modeled by two infinite parallel lines, separated by $2 a=2 \sqrt{A^{2}-R^{2}}$ and of uniform linear charge $\pm 2 \pi \epsilon_{0} V_{0} / \cosh ^{-1}(A / R)$.

Defining $\alpha=A / R$, the capacitance per unit length of those two cylinders is

$$
C=\frac{\pi \epsilon_{0}}{\cosh ^{-1}(\alpha)}
$$

and, as a TEM resonant line, its inductance per unit length and impedance will be

$$
\begin{aligned}
& L=\frac{\mu_{0}}{\pi} \cosh ^{-1}(\alpha), \\
& Z=\frac{Z_{0}}{\pi} \cosh ^{-1}(\alpha),
\end{aligned}
$$

where $Z_{0}=\left(\mu_{0} / \epsilon_{0}\right)^{1 / 2}$ is the vacuum impedance.

From the capacitance the energy content of the electromagnetic mode at a given voltage $\pm V_{0}$ at the center of the bars can be calculated:

$$
\begin{aligned}
U & =\int_{-\lambda / 4}^{\lambda / 4} \frac{1}{2} C V^{2} d z \\
& =\frac{1}{2} C\left(2 V_{0}\right)^{2} \int_{-\lambda / 4}^{\lambda / 4} \cos ^{2}\left(\frac{2 \pi z}{\lambda}\right) d z \\
& =\frac{\lambda}{2} C V_{0}^{2}=\frac{\lambda}{2} \frac{\pi \epsilon_{0}}{\cosh ^{-1}(\alpha)} V_{0}^{2} .
\end{aligned}
$$

The total power dissipation $P$ is made of two parts, $P_{b}$ the power dissipation on the two bars, and $P_{p}$ the power dissipation on the two planes. The power dissipation on the two bars is

$$
\begin{aligned}
P_{b} & =2 \times \frac{R_{s}}{2} \int_{\mathrm{bar}} H^{2} d S \\
& =R_{s} \int_{-(\lambda / 4)}^{\lambda / 4} \sin ^{2}\left(\frac{2 \pi z}{\lambda}\right) d z \int_{C} H^{2} d l=R_{s} \frac{\lambda}{4} \int_{C} H^{2} d l
\end{aligned}
$$

where the last integral is a linear integral over one of the circular intersections between the bars and the planes, and $R_{s}$ is the surface resistance of the cavity material.

Because of the relationship between electric and magnetic fields in TEM modes, the integral of $H^{2}$ over $C$ is equal to the integral of $\left(E / Z_{0}\right)^{2}$ over $C^{\prime}$, the circular intersection of one of the bars with the midplane where the voltage and electric field are maximum:

$$
\int_{C} H^{2} d l=\frac{1}{Z_{0}^{2}} \int_{C^{\prime}} E^{2} d l
$$

where $E^{2}=E_{x}^{2}+E_{y}^{2}$, which are given in Eqs. (A6) and (A7), and

$$
\begin{gathered}
x=A+R \cos \phi, \\
y=R \sin \phi .
\end{gathered}
$$

After some algebra, $E^{2}$ on $C^{\prime}$ is found to be

$$
E^{2}\left(C^{\prime}\right)=\frac{V_{0}^{2}}{R^{2}} \frac{\alpha^{2}-1}{\left[\cosh ^{-1}(\alpha)\right]^{2}} \frac{1}{(\alpha+\cos \phi)^{2}},
$$

and

$$
\begin{aligned}
\int_{C^{\prime}} E^{2} d l & =\frac{V_{0}^{2}}{R^{2}} \frac{\alpha^{2}-1}{\left[\cosh ^{-1}(\alpha)\right]^{2}} \int_{0}^{2 \pi} \frac{R d \phi}{(\alpha+\cos \phi)^{2}} \\
& =\frac{V_{0}^{2}}{R} \frac{2 \pi}{\left[\cosh ^{-1}(\alpha)\right]^{2}} \frac{\alpha}{\sqrt{\alpha^{2}-1}} .
\end{aligned}
$$

So the power dissipation on the bars is

$$
P_{b}=\frac{\pi}{2} \frac{\lambda}{R} \frac{R_{s} V_{0}^{2}}{Z_{0}^{2}} \frac{1}{\left[\cosh ^{-1}(\alpha)\right]^{2}} \frac{\alpha}{\sqrt{\alpha^{2}-1}} .
$$

The power dissipation on the two planes is

$$
P_{p}=2 \times \frac{R_{s}}{2} \int_{\{P\}-2\{D\}} H^{2} d S,
$$

where the integral is over the complement on one plane of the two disks that are the intersections of the bars with that plane. That integral might be lengthy to calculate directly but can be expressed simply in terms of the energy content since

$$
U=\frac{\mu_{0}}{2} \int_{V} H^{2} d V=\frac{\mu_{0}}{2} \frac{\lambda}{4} \int_{\{P\}-2\{D\}} H^{2} d S .
$$

This implies, from Eq. (A14),

$$
\int_{\{P\}-2\{D\}} H^{2} d S=\frac{8 U}{\lambda \mu_{0}}=\frac{4 \pi}{Z_{0}^{2}} \frac{V_{0}^{2}}{\cosh ^{-1}(\alpha)},
$$

and

$$
P_{p}=4 \pi \frac{R_{s} V_{0}^{2}}{Z_{0}^{2}} \frac{1}{\cosh ^{-1}(\alpha)} .
$$


The total power dissipation is then

$$
P=\frac{\pi R_{s} V_{0}^{2}}{Z_{0}^{2}} \frac{1}{\cosh ^{-1}(\alpha)}\left[\frac{\lambda}{2 R} \frac{1}{\cosh ^{-1}(\alpha)} \frac{\alpha}{\sqrt{\alpha^{2}-1}}+4\right] .
$$

The transverse voltage acquired by an on-crest particle is simply the integral of the time-dependent transverse electric field along the beam line. In an actual structure, end reflecting walls will be located at $y= \pm \lambda / 4$ so the fields will not extend further. The present simple model, where $y$ extends from $-\infty$ to $+\infty$, can be made more realistic by including all the images of the charged lines created by the two reflecting walls. The transverse electric field along the beam line is then

$$
\bar{E}_{x}(y)=\sum_{k=-\infty}^{k=\infty}(-1)^{k} E_{x}(0, y+k \lambda / 2),
$$

where, from Eq. (A6),

$$
E_{x}(0, y)=\frac{a q}{\pi \epsilon_{0}} \frac{1}{a^{2}+y^{2}}
$$

The transverse voltage is then

$$
\begin{aligned}
V_{t} & =\int_{-\lambda / 4}^{\lambda / 4} d y \bar{E}_{x}(y) \cos \omega t \\
& =\sum_{-\infty}^{\infty} \int_{-\lambda / 4}^{\lambda / 4} d y(-1)^{k} E_{x}\left(0, y+\frac{k \lambda}{2}\right) \cos \left(\frac{2 \pi y}{\lambda}\right) \\
& =\int_{-\infty}^{\infty} d u E_{x}(0, u) \cos \left(\frac{2 \pi u}{\lambda}\right) \\
& =\frac{a q}{\pi \epsilon_{0}} \int_{-\infty}^{\infty} \frac{d u}{a^{2}+u^{2}} \cos \left(\frac{2 \pi u}{\lambda}\right)=\frac{q}{\epsilon_{0}} \exp \left[-\frac{2 \pi a}{\lambda}\right] \\
& =V_{0} \frac{2 \pi}{\cosh ^{-1}(\alpha)} \exp \left[-2 \pi \frac{R}{\lambda} \sqrt{\alpha^{2}-1}\right] .
\end{aligned}
$$

Equation (A29) indicates that, if the reflecting walls are located exactly $\lambda / 4$ on either side of the center of the cavity, the transverse voltage obtained by including all the images is identical to that obtained when those walls are absent.

Expressed in terms of the transverse voltage $V_{t}$ or the transverse field $E_{t}=2 V_{t} / \lambda$, the energy content is

$$
\begin{aligned}
U & =\frac{\epsilon_{0}}{8 \pi} \lambda V_{t}^{2} \cosh ^{-1}(\alpha) \exp \left[4 \pi \frac{R}{\lambda} \sqrt{\alpha^{2}-1}\right] \\
& =\frac{\epsilon_{0}}{32 \pi} \lambda^{3} E_{t}^{2} \cosh ^{-1}(\alpha) \exp \left[4 \pi \frac{R}{\lambda} \sqrt{\alpha^{2}-1}\right] .
\end{aligned}
$$

From the energy content $U$, the power dissipation $P$ and the transverse voltage $V_{t}$, the quality factor $Q=\omega U / P$, the geometrical factor $G=Q R_{s}$, and $R_{t} / Q=V_{t}^{2} / P$ can be easily calculated.
[1] A. Citron, G. Dammertz, M. Grundner, L. Husson, R. Lehm, H. Lengeler, D. Plane, and G. Winkler, Nucl. Instrum. Methods 155, 93 (1978).

[2] H. Padamsee, P. Barnes, C. Chen, J. Kirchgessner, D. Moffat, D. Rubin, Y. Samed, J. Sears, Q.S. Shu, M. Tigner, and D. $\mathrm{Zu}$, in Proceedings of the 1991 Particle Accelerator Conference, San Francisco, California (IEEE, Piscataway, NJ, 1991), p. 2423, Catalog No. 91CH3038-7.

[3] K. Hosoyama, K. Hara, A. Kabe, Y. Kojima, Y. Morita, H. Nakai, L. S. Peng, K. Ohkubo, H. Hatori, and M. Inoue, in Proceedings of the 1998 Asian Particle Accelerator Conference, Tsukuba, Japan, KEK Proceedings 98-10 (KEK, Tsukuba, Japan, 1998), p. 828.

[4] T.W. Koeth, in Proceedings of the 1999 Particle Accelerator Conference PAC'09, New York (IEEE, Piscataway, NJ, 1999), p. 995, Catalog No. 99CH36366.

[5] C. W. Leemann and C. G. Yao, in Proceedings of the 1990 Linear Accelerator Conference LINAC'90, Albuquerque, New Mexico (Los Alamos Report No. LA-12004-C, 1991), p. 232.

[6] Y. Senichev, O. Belyaev, W. Bräutigam, R. Maier, V. Tepanov, V. Teplyakov, A. Zherebtsov, and I. Zvonarev, Phys. Rev. ST Accel. Beams 9, 012001 (2006).

[7] C. Hovater, G. Arnold, J. Fugitt, L. Harwood, R. Kazimi, G. Lahti, J. Mammosser, R. Nelson, C. Piler, and L. Turlington, in Proceedings of the 1996 Linear Accelerator Conference LINAC'96, Geneva, Switzerland, 1996 (CERN Report No. 96-07, 1996), p. 77.

[8] R. B. Palmer, SLAC Technical Report No. SLAC-PUB4707, 1988.

[9] K. Oide and K. Yokoya, Phys. Rev. A 40, 315 (1989).

[10] A. Zholents, P. Heimann, and M. Zolotorev, Nucl. Instrum. Methods Phys. Res., Sect. A 425, 385 (1999).

[11] J. Shi, H. Chen, S. Zheng, J.M. Byrd, and D. Li, in Proceedings of the 2005 Particle Accelerator Conference PAC'05, Knoxville, Tennessee (IEEE, Piscataway, NJ, 2005), p. 350, Catalog No. 05CH37623C.

[12] J.R. Delayen, in Proceedings of the Tenth Workshop on RF Superconductivity, Tsukuba, Japan, 2001, KEK Proceedings 2003-2 (KEK, Tsukuba, Japan, 2003), p. 152.

[13] J. R. Delayen, M. Spata, and H. Wang, in Proceedings of the 2009 Particle Accelerator Conference PAC'09, Vancouver, BC, Canada, 2009.

[14] B. Hall, G. Burt, J.D. A. Smith, R. Rimmer, H. Wang, J. Delayen, and R. Calaga, in Proceedings of the 2009 Particle Accelerator Conference PAC'09, Vancouver, BC, Canada, 2009.

[15] R. Calaga, U. Dorda, R. Tomás, F. Zimmermann, K. Akai, K. Ohmi, and K. Oide, in Proceedings of the 2007 Particle Accelerator Conference PAC'07, Albuquerque, NM (IEEE, Piscataway, NJ, 2009), p. 1853, Catalog No. $07 \mathrm{CH} 37866$.

[16] R. Calaga, Y. Sun, R. Tomás, and F. Zimmermann, in Proceedings of the 11th European Particle Accelerator Conference, Genoa, 2008 (EPS-AG, Genoa, Italy, 2008).

[17] J. R. Delayen, Physica C (Amsterdam) 441, 1 (2006).

[18] K. Ko et al., Physica C (Amsterdam) 441, 258 (2006). 